\title{
Managing PV power injection and storage, enabling a larger direct consumption of renewable energy \\ A case study for the Belgian electricity system.
}

\author{
Marc Meuris ${ }^{1,2,3}$, Pieter Lodewijks ${ }^{1,4}$, Raf Ponnette ${ }^{1,4}$, Frank Meinke-Hubeny ${ }^{1,4}$, Pieter \\ Valkering $^{1,4}$, Ronnie Belmans ${ }^{1,5}$, and Jef Poortmans $s^{1,3,5,6}$. \\ ' EnergyVille, Thorpark 8310-8320, B-3600 Genk, Belgium \\ 2 imec division IMOMEC, Wetenschapspark I, B-3590 Diepenbeek, Belgium \\ ${ }^{3}$ Institute for Material Research (IMO), Hasselt University, Wetenschapspark I, B-3590 Diepenbeek, Belgium \\ ${ }^{4}$ VITO, Boeretang 200, B-2400 Mol, Belgium \\ ${ }^{5}$ Department of Electrical Engineering (ESAT), KU Leuven, Kasteelpark Arenberg 10, B-300I Heverlee, \\ Belgium \\ ${ }^{6}$ imec, Kapeldreef 75, B-300I Leuven, Belgium
}

Corresponding author: Marc Meuris, EnergyVille, Thorpark 8320, B-3600 Genk, Belgium, marc.meuris@imec.be, +3216281350

\begin{abstract}
: (max. 250 words)
An overall model and scenario for increasing the share of renewable electricity consumption in the Belgium grid is proposed by simulating different PV and home battery capacities. The model is balancing the electricity generation of these renewable sources on an hourly basis with the historical consumption profiles of 2014, 2015 and 2016 to estimate how much renewable energy can be consumed directly. The model considers the problem of potential overloads on the low voltage grid, by limiting with curtailment the total PV injection on the low voltage grid to a level that can be currently sustained. A simple algorithm for grid injection limitation of this curtailment during day and night time is proposed, with and without the use of batteries. The share of renewable electricity consumption is calculated, investigating the impact of battery sizing and different levels for the power injection limit. There is an upper limit beyond additional battery capacity has no effect. With increasing total PV deployment, the optimal power injection limit is reduced. A realistic and effective deployment scenario for renewable electricity from wind and PV is developed (up to $9 \mathrm{GW}$ wind and $50 \mathrm{GWP}$ PV for the Belgian case). It is possible to reach a share of consumed renewable electricity of almost $50 \%$ with a curtailment of $9 \%$ without the use of batteries. With higher PV deployment and a minimal amount of home battery capacity ( 1.5 $\mathrm{kWh} / \mathrm{kWp}$ ) a $70 \%$ share of consumed renewable energy is realized, with a curtailment of less than $20 \%$.
\end{abstract}

Keywords: (max. 6)

Curtailment

grid injection limit

distributed storage system

self-consumption

direct consumption

Photovoltaics (PV) 


\section{Introduction}

Climate change is now widely accepted as a fact by the scientific community. Most solutions proposed by political and industrial decision makers entail a major shift of the existing electricity generation technologies, mainly based on fossil fuels towards renewable energy generation techniques [1], [2]. An excellent motivation can be found in [3], underlining that for renewable electricity, the best options are wind and solar as the most cost-effective renewable energy, apart from more conventional hydropower. These findings are confirmed by current market developments, indicating that solar and wind are currently the fastest growing renewable energy sources globally [4],[5],[6]. This study focuses on the potential of wind and solar energy for transforming the Belgian electricity system towards renewables at a minimal societal cost. In 2016, power generation in Belgium is to $55 \%$ provided by nuclear power plants while solar PV and onshore and offshore wind account for I4\% [7]. Current Belgian policy [8] is committed to both an energy transition towards a low carbon society in 2050 , as well as the complete retirement of the $5.9 \mathrm{GW}$ nuclear generation capacity planned in the period 2020 to 2025 . To replace nuclear capacity, significant growth of the renewable based generation can be expected with roughly equal shares of renewable and fossil fuel based electricity generation in a cost-optimal scenario for 2030 [7]. Despite its legal status, the nuclear phase out is still subject to political and societal debate. However, from the cost optimization perspective a possible nuclear extension up to $2 \mathrm{GW}$ capacity would up to 2030 replace mainly fossil fuel based generation, with only a minor impact on renewable based generation.

The main issue using these renewable resources is their intermittency. The electricity consumption and the generation profile of wind and solar energy will not match each time. Many studies have investigated the potential of shifting the consumption (or demand) towards the generation profile of the renewable resources, by demand side response [9]; [10]. However, this will also have its limitations regarding the overall flexibility potential, in particular, for non-automated applications. Part of the consumption can be shifted, but for substantial shifts, it requires a relative high reward or price benefit for the consumer [9], making the current business cases for the residential market difficult to realize [I I]; [I2]. Moreover, a suite of economic, social, technical, political and regulatory barriers to demand response still need to be overcome [13]. For the large deployment of renewables into the electricity system, we need to maximize the potential of direct consumption of the renewable generated energy as a complementary approach to demand response. This requires rethinking how energy storage can support direct consumption in the most costeffective ways.

When wind and solar generated energy is fed directly into the electricity grid, then cost is already competitive with existing electricity generation based on fossil fuels [14]. In principal, when electricity storage is available at low additional cost, wind and solar energy is competitive for most of the electricity generation. Batteries, being the principal distributed electrical energy storage, still add a significant extra cost to the electricity system [15];[16], despite the present and future expected price decline [17],[18],[19]. In [20], a study can be found assessing a high penetration level of PV generation and battery storage in Germany. In [2I], the cost of self-sufficiency for a PV-battery system is calculated in detail for the Belgian electricity regulatory framework. Typical price levels of 30 to $40 \mathrm{c} € / \mathrm{kWh}$ are derived for self-sufficiency levels of $70-80 \%$. Fares and Webber [22] point out that battery storage may 
increase greenhouse emissions due to storage inefficiencies, unless it directly enables renewable energy that otherwise would have been curtailed. Green and Staffel [23] highlight the economic limitations of household scale storage in the British energy market, both for the business cases of arbitrage and self-consumption, arguing that more complex (and hence unattractive) business cases would be needed to make household battery storage economically viable.

Most studies addressing the combination of a PV and a battery system, based on the concept of self-consumption on a local or house scale and assess the cost-effectiveness of such a combination. However, battery storage makes this solution still relatively expensive, compared to the traditional electricity generation technologies [2I]. A system with subsidies is required to enable a business case for the combination of PV and battery storage to realize self-consumption for houses, or alternatively, more complex business models for batteries are needed [23]. Moreover, self-consumption as the only measure is not sufficient to reach a $100 \%$ renewable energy supply in the residential sector based on PV in Belgium and surrounding regions. $100 \%$ self-consumption would require a very uneconomic set-up with unrealistic large battery and PV sizing [23]. With more realistic numbers of sizing the PV and battery system, already a high ratio of self-consumption may be attained [2I]. During the periods in which the system does not provide sufficient power, it is most convenient to use the electricity grid. But, it would be difficult to find a cost-effective business model for the electricity grid infrastructure with many high self-sufficiency consumers, due to the limited time the grid infrastructure is used by these consumers.

\section{Scope}

A modeling case study, evaluating a combination of battery storage and PV to support the PV injection on the Belgian electricity grid, is presented. The primary aim is not to increase self-consumption, but to obtain the highest possible direct consumption of the generated wind and solar power electricity in the overall Belgian electricity system. The battery is only added to capture the solar energy, which cannot be consumed instantaneously. The battery is discharged, not for self-consumption, but at the moment it does not coincide with injection from the PV system, i.e. during each night, just after the battery has been loaded during day time. In this way, the injection of renewable power is ensured, whereby direct injection is the most cost-effective use of the renewable energy. The modeling is based on the historical data of PV and wind availability factors per hour and the real Belgian electricity consumption per hour over the years 2014, 2015 and 2016. By simulating different wind and PV power capacities, the injection of renewable power and curtailed energy is calculated on an hourly basis, under the constraint of matching demand and supply. The PV and wind power capacities are chosen to maximize the injection of the generated renewable electricity. The historical consumption data are used and no demand side management is assumed. Therefore, the results may be considered as worst-case results, whereby extra measures could still increase the use of renewable energy. By increasing the wind and PV power capacities, inevitably, some curtailment is needed to match the consumption profile. In this study, a curtailment of $10 \%$ is allowed, before batteries are introduced in the calculations. In the foreseeable future, this moderate curtailment is considered as more cost-effective than adding battery storage, because such a curtailment would only add $10 \%$ to the cost of wind and PV energy. An optimal sizing of the battery storage capacity is then calculated to increase the share of renewable energy consumed in the system and to allow a moderate curtailment. A simple and transparent load/unload algorithm for the battery is proposed, ensuring a long-life time for the battery. This algorithm could be optimized 
further, but that should be the scope of other studies. For countries, with a comparable moderate climate to Belgium, a similar approach can be adopted to increase the use of renewable electricity at minimal costs for grid upgrades.

\section{Grid injection limitation for the PV systems}

Currently (status in 2016), the installed base in Belgium for PV is about $3 \mathrm{GWP}$ of PV systems, of which $2 \mathrm{GWp}$ is located on residential buildings [7]. For offshore wind it is 0.7 GW and I.5 GW for onshore [7]. When at a certain time all renewable installations would generate at full capacity, 5.2 GW renewable power could be injected in the Belgian electricity grid. Theoretically, the other sources of electricity generation may be dispatched to enable all renewable electricity to be consumed directly, as the Belgian electricity consumption never drops below $5.5 \mathrm{GW}$ [24]. This is possible for the generation by offshore wind connected to the high voltage $(\mathrm{HV})$ grid and onshore wind, connected to the high and medium voltage (MV) grid. Therefore, wind energy can be distributed across the complete grid. For the PV systems, $2 \mathrm{GWP}$ (i.e. the residential share) is connected to the low voltage (LV) grid., Already today, in rare cases this causes local congestion problems. When the voltage exceeds locally a critical level, typically a switch-off of the PV system from the grid is used to protect the electricity grid, causing an unpredicted loss of renewable generated power. With increasing penetration, this congestion will occur more and local reinforcements in the LV grid may be needed. A control algorithm for limiting the injection into the LV grid of the PV generated power could help to avoid such local overload problems on the LV grid.

The Belgian electricity system is designed to allow on every connection in the grid a continuous consumption of about $1.5 \mathrm{~kW}$. There are about 5.8 million connections on the LV grid in Belgium [25]. This means that theoretically about $8.7 \mathrm{GW}$ can be continuously transported on the LV grid, limiting the total amount of power that from residential PV installations can be injected into the grid. If more than $8.7 \mathrm{GWP}$ of residential solar would be installed and every installation has an invertor of I $\mathrm{kW} / \mathrm{kWP}$, then at certain times the LV grid would be overloaded. However, in practice many professional installers found out heuristically that invertors of 0.7 to $0.9 \mathrm{~kW} / \mathrm{kWP}$ generate almost the same annual energy compared to larger sized invertors. In Flanders, it becomes a common practice to limit the invertor to about $0.7 \mathrm{~kW} / \mathrm{kW}$, due to the prosumer tax based on the invertor power capacity. Even then, only a limited amount of time the invertors operate at full capacity.

Fig. I shows the normalized annual energy yield (in $\mathrm{kWh} / \mathrm{kW}$ ) for the power (in $\mathrm{kW}$ ), generated by $\mathrm{I} \mathrm{kW}$ installation, distinguishing 4 different orientations (Direction/inclination: S-30: South $/ 30^{\circ}, \mathrm{H}-00$ : Horizontal $/ 0^{\circ}, \mathrm{S}-90$ : South $/ 90^{\circ}$ and W-30: West $/ 30^{\circ}$ ). Most of the annual energy is generated below $0.5 \mathrm{~kW}$ for a I $\mathrm{kWp}$ installation. Therefore, an algorithm is proposed to limit the power injection into the grid to a certain fixed maximum injection limit per installed kWP of PV. In Fig 2, a schematic is shown how it works. If the PV system is not reaching the limit, all the power is injected in the grid. If the system generates more power than the injection limit, only the power below the power injection limit is sent to the grid. The part above is curtailed.

This type of injection limit has already been studied, introduced and discussed in Germany [26]; [27]; [28]. However, the purpose of these studies was mainly to reduce local congestion and to stimulate self-consumption with battery storage. The aim of this study is not to optimize the PV/battery system set-up for increasing the self-consumption, but to 
increase the share of renewable energy in the total Belgian electricity consumption and at the same time to keep the LV power injection below the design limit of the LV grid. An optimal amount of battery storage to accompany the PV installations for this purpose is simulated.

Assuming more and more PV systems are installed in the Belgian LV grid, a grid injection limitation for each installed $\mathrm{kWp}$ could drastically reduce the need of reinforcing this LV grid. Based on the design rule of $1.5 \mathrm{~kW}$ per connection point, a limitation of $0.5 \mathrm{~kW}$ per installed kWp, would allow roughly up to $17.4 \mathrm{GWp}$ to be placed in the LV grid. Locally some upgrades would be needed, because the local distribution of the injected power will not perfectly match the local consumption in the district and the design of the LV grid. But the grid injection limit for each PV installation will avoid the need to go above the existing design limitation of the grid. Furthermore, a lower injection limit of e.g. 0.2 to 0.25 $\mathrm{kW} / \mathrm{kWp}$ could allow a deployment of roughly $30 \mathrm{GWP}$ with only these local and limited upgrades on the grid.

As a trade-off, an injection limit, leads to a loss of PV power, generated above the injection limit. In Fig. 3 (left) the amount of annual electrical energy, injected in the grid, for injection limits between 0.1 and $\mathrm{I} \mathrm{kW} / \mathrm{kWP}$ is calculated for the most effective orientation in Belgium (S-30: South oriented $-30^{\circ}$ inclined). The irradiation is based on a standard solar year at the site of Uccle, Belgium (irradiation data with a 15' interval). The orientation of S-30 generates an annual energy yield of about $1050 \mathrm{kWh}$. In Fig. 3 (right) the fraction of lost (not injected) energy of the PV generated annual energy (as a percentage of the maximum possible energy yield at I $\mathrm{kW} / \mathrm{kW}$ injection level) is calculated. At $0.5 \mathrm{~kW} / \mathrm{kWp}$, the annual energy loss for this orientation is $12 \%$ [29]. For all the other orientations there is less loss, because these orientations have less favorable conditions for high power generation levels. E.g., for a I $\mathrm{kW}$ installation in the horizontal plane in Belgium, the condition of I kW generation can never occur, because the sun position is never vertical in Belgium. So, one can consider the Fig. 3 (right) as a worst case.

Limiting the injection power of PV systems, allows to deploy more PV systems in the grid without congestion in the local LV grid, rejecting some power generated by PV. The cost of electricity from the PV system raises linearly with the amount of curtailed PV. From a cost perspective, rejecting 10 to $20 \% \mathrm{PV}$ generated power, is still more attractive now, when it leads to a larger use of renewable energy (less $\mathrm{CO}_{2}$ generation), than storing this surplus in a costly battery system. Also, other in-home direct uses of excess power may be applicable (e.g. for heating or car charging) which would further improve the business case, as further elaborated on in the discussion section. Table I shows the proposed grid injection limit as function of total installed PV capacity. In this Table, it is assumed that the limit of the Belgium grid for the total PV power injection is less than $6 \mathrm{GW}$, to stay more than $25 \%$ underneath the $8.7 \mathrm{GW}$ design rule for the continuous power on the LV grid in Belgium. This $6 \mathrm{GW}$ limitation will keep the local congestion problems to some rare cases and will minimize the upgrading costs for the LV grid. From this assumption, we adjust the power limitation for the PV systems when installations would increase from 5 to $60 \mathrm{GWP}$. The power injection limit is then determined by the total amount of PV installations. In this case all PV systems could inject on the low voltage grid if appropriate.

The maximum rejected power is based on a theoretical calculation for the best orientation (S-30) and provides an upper limit. When deploying more and more residential solar power, a lot of PV systems are also placed in different orientations (e.g. horizontal and between 
East-West with $25^{\circ}$ to $45^{\circ}$ degrees inclination), the real energy loss for such systems is lower than calculated in Table I. Moreover, even for the best orientation (S-30) the calculation for rejected energy is too high, because it does not consider losses in the inverter. The invertor efficiency is typically $97 \%$ to $99 \%$ between 0.1 and I of the rated power. Below 0.1 rated power, the conversion efficiency drops very fast. In the range of 0 to 0.1 rated power, about $7-8 \%$ of the total annual energy is generated (Fig. I). By lowering the maximum power of the invertor to e.g. $0.5 \mathrm{~kW} / \mathrm{kWp}$, part of this energy in this range is converted more efficiently. Therefore, lowering the injection limit and decreasing the invertor power at the same time, results in less losses than calculated in Table I for realistic PV systems. Due to different invertor specifications, it is difficult to predict the exact reduction in the energy lost, but one can estimate that a few percent of rejected electricity can be regained by using a power invertor of $0.5 \mathrm{~kW} / \mathrm{kWp}$ or smaller for the PV residential systems. This effect is not used in the calculations, but when a detailed quantitative analysis is assessed, it may not be neglected.

\section{Reduction of rejected power by local electrical storage}

In Table I, with more PV systems coupled to the grid, a larger portion of the generated power is rejected and may be stored in a battery system. E.g. if $20 \mathrm{GWp}$ is installed, a power injection limit of $0.3 \mathrm{~kW} / \mathrm{kW}$ is necessary to protect the LV grid and about $30 \%$ of the surplus generated PV electricity is rejected. This electric energy may be stored in a home battery and during the night injected in the grid. In Belgium, typically consumption power at day time is around 9 to $12 \mathrm{GW}$ and during night time 6 to $8 \mathrm{GW}$. An injection during day time of $6 \mathrm{GW}$ from the solar power and during night time of 2 to $3 \mathrm{GW}$ from the battery allows wind power to add 3 to $6 \mathrm{GW}$ before curtailment on wind power will take place. Although storing the surplus energy of a solar system seems an obvious decision, the cost of adding batteries is substantial. While several authors [20];[21];[22] suggest a $\mathrm{PV} /$ battery system combination for self-consumption, in this study it is suggested to use the battery only to store power of the PV system, rejected by the grid, during day time and unload the battery only during night time by injection in the grid, when the PV system is not generating energy.

In the model, a certain fixed amount of battery capacity $(\mathrm{kWh})$ is installed per $\mathrm{kWp}$ of solar panels. Batteries are installed behind the meter, to share the DC/AC invertor, so only one $D C / A C$ invertor is needed. This assumption also ensures that the excess electricity generated by the PV system and rejected by the power injection level of the grid, does not enter the LV grid, but is stored at the home side. If the battery is not placed behind the meter, but somewhere in the district, locally the grid can still be overloaded, based on the $1.5 \mathrm{~kW}$ per connection design limit of the Belgian grid. The algorithm assumes that for all batteries, the charging process from the solar panel, when generating more than the injection limit, is equal. It has no influence on the grid, as the battery is placed behind the meter.

In Fig. 4 a simple algorithm is proposed. In Fig. 4 (top) the solar panels deliver less than the maximum injection level. The battery is not used and all power is injected in the grid during day time. In Fig. 4 (bottom), the algorithm is shown for the charging and discharging of the battery. The battery will be loaded, when the PV power reaches the maximum injection level. When the battery is full, the extra electricity from the solar panels above the injection limit is rejected, leading still to some losses of the annually generated PV energy. In the evening, at $2 \mathrm{lh}$, when the solar panels generate almost no power, the batteries are 
programmed to inject their energy in the grid. This night injection level is fixed for the full unload cycle during the night. This level is low enough to not reach the maximum amount of consumption on the Belgian grid during the night. Also, wind energy can be injected during the night. The level is high enough to empty the battery during a period of maximum IOh. This ensures a fresh empty battery in the morning to be recharged, once the PV systems inject power again. In the simulation, the night injection starts at $2 \mathrm{Ih}$ and stops at $7 \mathrm{~h}$ in the morning. The power injection level is chosen to empty the battery in these IOh. The time interval of 10 hours is chosen to avoid that the day injection from the PV system itself coincides with the night injection from the battery. For Belgium, that may occur in summer before $2 \mathrm{Ih}$ and after $7 \mathrm{~h}$. For regions on another latitude, these hours should be adapted. The standard time in Belgium is used and daylight savings time is not used.

In Table II, the night injection level is shown as function of the net battery capacity. A round trip efficiency of $90 \%$ for the net battery capacity is assumed [15];[16];[29]. Therefore, the battery will inject during night time only $90 \%$ of its energy accumulated during day time. A I $\mathrm{kWh}$ net battery injects during the night $0.9 \mathrm{kWh}$, and therefore the power injection limit is $0.09 \mathrm{~kW}$ in $10 \mathrm{~h}$. If the night injection is not higher than $0.15 \mathrm{~kW}$ per $\mathrm{kWP} P V$ installed, PV with battery can be deployed up to $40 \mathrm{GWP}$ of total solar capacity, hooked up to the LV grid, without exceeding the $6 \mathrm{GW}$ total injection limit on that LV grid. For a $2 \mathrm{kWh} / \mathrm{kWp}$, battery added to the PV installation in the case of $40 \mathrm{GWP}$ deployment, this cannot be fulfilled. A combination of more than $34 \mathrm{GWp}$ with $2 \mathrm{kWh} / \mathrm{kWp}$ battery (night injection level of $0.18 \mathrm{~kW} / \mathrm{kWP}$ ) results in a larger than $6 \mathrm{GW}$ injection on the LV grid.

The battery capacity is used as a net capacity. In a real PV/battery system, the Depth of Discharge (DoD) of most $\mathrm{Li}$ ion batteries should not exceed 70 to $80 \%$ of the full capacity to ensure many load/unload cycles. For cost estimates, it is important to add this $20-30 \%$ extra battery capacity. When a net battery capacity of $\mathrm{I} \mathrm{kWh} / \mathrm{kWp}$ is simulated, in practice, 1.2 to $1.3 \mathrm{kWh} / \mathrm{kWp}$ total capacity must be installed.

The choice of the power levels for the loading/unloading algorithm in this study ensure that the battery gets a maximum of I load/unload cycle per day. Combined with the choice of a $75 \%$ DoD, it ensures a lifetime of minimally 20 years for properly chosen Li-ion battery systems. In practice, even life times of 30 years are feasible [30],[3 I], [32]. In the simulations, using the proposed algorithm, the typical amount of one load/unload cycle only happens between 150 days per year (at $0.5 \mathrm{~kW} / \mathrm{kWP}$ PV injection level) and 250 days per year (at $0.15 \mathrm{~kW} / \mathrm{kWp}$ PV injection level). In the remaining 200 days (at $0.5 \mathrm{~kW} / \mathrm{kWp}$ PV injection level) and 100 days (at $0.15 \mathrm{~kW} / \mathrm{kWP}$ PV injection level) respectively, the PV system injects all generated electric energy directly in the grid. Even with very low power injection limits, the battery is only used for about 250 load/unload cycles a year. This limits the load/unload cycles to about 7500 cycles over 30 years, i.e. within the life time specifications of some Li-ion batteries already now [15], making the battery proposal algorithm economically attractive.

Another reason to choose this 'mild' algorithm for the battery is that the DC/AC invertor always operates between $10 \%$ and $100 \%$ of its rated power, minimizing power conversion losses. Using this algorithm, whereby the battery is never unloaded below a DoD of $75 \%$ and whereby the DC/AC conversion is never below $10 \%$, one can estimate that the battery round trip efficiency is $85-90 \%$. In this study, a roundtrip efficiency of $90 \%$ is used. 


\section{Methodology of the modeling}

To find out how much renewable energy with a minimal amount of battery storage can be used on the Belgian electricity grid, the potential renewable energy generation is modeled and compared to historical data of the electricity consumption. For the years 2014, 2015 and 2016, the total Belgium consumption of electric power and the availability factors of PV, offshore wind and onshore wind per hour were used [33]. The availability factor is a normalized number that on an hourly basis gives the \% availability of the total installed capacity of an electric generation system. As all power of renewable resources has priority on the Belgian electricity grid, this number is used to estimate how much renewable power of PV and wind is available per hour over the whole year. In the model, this number is taken and multiplied by the simulated capacity per renewable energy source to define on an hourly base how much renewable power can be generated by deploying more PV or wind capacity. In the model, 4 power generators are present: PV, offshore wind, onshore wind and gas. It reflects the assumptions of a report of the Belgian planning office [33], whereby the deployment of PV and wind in the Belgian electricity grid is modeled based on wind, PV and gas. In [24], battery storage is not considered and a renewable electricity generation between 50 and $60 \%$ is assumed to be achievable. In the model, gas can also be substituted by any other power source, which can react sufficiently fast on the intermittency of PV and wind. E.g. import of electricity (when available) could also be a source of electricity to complement the energy mix, but for this study it is not important, because we want to simulate how much electricity can be consumed from the PV and wind. The gas component can be considered as any other non-renewable source, complementing the renewable resources. It is a worst-case scenario for renewable electricity use, because if gas is replaced by import, part of the imported electricity may also come from renewable resources. It is difficult to predict how much this will be in the future. Therefore, only the PV and wind are considered as renewables.

Before using the model, it is checked if the availability factor for PV injection on the Belgian grid [24] is similar to the theoretical calculated generation of a PV system. Fig. 5 shows the annual frequency of the power injection level of the availability factor for the years 2014, 2015 and 2016 of a I kWP PV system (i.e. the availability factor is multiplied by I kW). Besides some small variations, the three profiles are very close as expected in the moderate climate of Belgium. This is compared to a theoretically calculated hourly frequency from a S30 oriented PV system. Also, this curve is very close to the real-time data, but in this case more hours of 0.8 and $0.9 \mathrm{~kW}$ power generation are predicted than realized in the availability factor during the years 2014,2015 and 2016 . This can be explained by the practice of limiting the invertor power to about 80 or $90 \%$ of the rated $k W_{P}$ on many PV systems, resulting already now in a practical limit of the PV injection power.

In the simulation, a power injection limit on the PV availability factor is introduced, changing the value of this factor, using the algorithms described in Fig. 2 and Fig. 4. The injection limit is preset based on the total amount of installed PV, according to Table I. It ensures always a PV power injection of maximum $6 \mathrm{GW}$ on the Belgian LV grid. If the PV availability factor for a certain hour is higher than the value of the power injection limit, the injection factor is set to the value of the power injection limit. When no battery is used, the surplus energy (above the injection limit) is summed over a year and labeled as lost. With a battery system, the surplus energy is stored daily into the battery, until it is full. The PV power above the power injection limit when the battery is full, is counted as lost. The energy stored in the 
battery, is daily injected in the Belgian grid from $2 \mathrm{Ih}$ onwards, at a night injection limit, dependent on the net battery capacity (Table II). This night injection limit is set as the injection factor until the battery is empty (maximum of IOh). This adapted injection factor is used for the PV part of the renewable power.

Total renewable energy curtailment is calculated as follows. The renewable energy conditions are varied by setting a certain amount of power capacity for PV, offshore and onshore wind. For every hour of the year, these capacities are multiplied with the corresponding injection and availability factors. For each hour, the generated energy of these three renewable energy sources is summed and the sum is compared with the Belgian electricity consumption. If it is less than the consumption, all renewable electric energy is consumed. If the renewable generated energy is higher than the consumption, the contribution to the renewable energy equals the consumed energy of that hour and the surplus is counted as lost. PV curtailment is counted by the power injection limit algorithm, while wind curtailment is counted when the renewable energy exceeds the consumption. Other curtailment algorithms are possible, but are not relevant for this study of maximizing the total amount of consumed renewable energy. At no point in time, the PV injected electricity is allowed to exceed the electricity consumption.

For each hour of the year $(2014,2015,2016)$ the generated renewable energy electricity (REE) is calculated with following model:

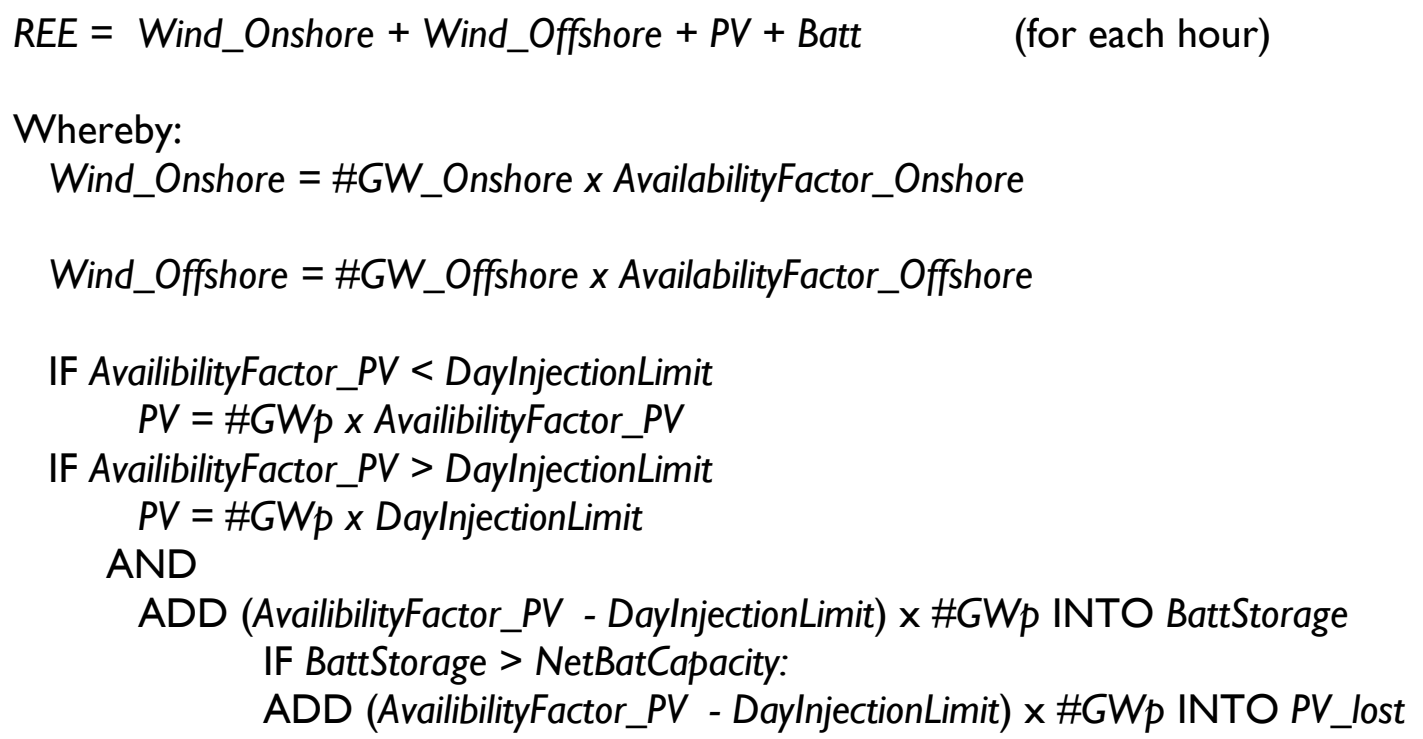

FROM 2Ih UNTIL 7h: Batt $=\# G W p \times$ NightInjectionLimit UNTIL BattStorage $=0$

FROM 7h UNTIL 2Ih: Batt $=0$

These values are calculated for each hour of a year and balanced with the consumption per hour of that year $(2014,2015$ or 2016)

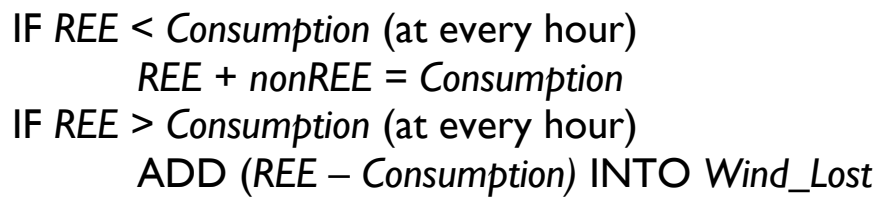

In this way, the generated power matches exactly with the consumption for each hour of the year. This ensures a perfect match between consumed and generated power. After the 
simulation of one set of offshore wind, onshore wind, PV and battery power capacity, the consumed (i.e. injected in the grid) renewable energy (in GWh) is summed up for a whole year as well as the lost renewable energy (in GWh). The injected renewable energy is divided by the total energy consumption to get the share of the renewable energy over the total consumed energy that year. The lost energy is divided by the total generated renewable energy to get the fraction of the curtailed renewable energy. The purpose of the simulation study is to maximize the renewable energy consumed and to minimize the fraction of curtailed renewable energy.

For each year of 2014, 2015, 2016, we calculated two parameters SRE and FRC:

- SRE: Share of renewable electricity in total consumption

- FRC: Fraction of renewable electricity curtailed over renewable energy production

Whereby:

SRE: = Sum_all_hours (REE) / Sum_all_hours (Consumption)

FRC $=$ Sum_all_hours $\left(P V \_l o s t+\right.$ Wind_lost $) /$ Sum_all_hours (generated_REE)

When the REE is lower than the present consumption of that hour, the energy needed to balance grid consumption is added to a counter called nonREE, being any power plant that can cope with the variations of the intermittent wind and PV generated electricity, i.e. gas, import or another energy source, but it is not the scope of this study. Also export of curtailed renewable energy is not taken into account and therefore the results can be considered as a worst-case scenario for the use of renewable energy. I.e. the surplus of wind energy can be exported through the HV grid to the neighboring countries. Extra degrees of freedom exist to increase the share of renewable energy consumed and this is discussed in the discussion section.

\section{Results of the model}

\section{Share of renewable energy for different PV/battery capacity combinations}

The historical data of the renewable energy availability factors in 2015 are first used to simulate a variety of renewable power generation capacities. In the appendix, the findings of the data of 2015 are validated with the data for 2014 and 2016, showing the sensitivity towards yearly weather variations.

For the generation from wind, a capacity for onshore of $5 \mathrm{GW}$ and offshore of $4 \mathrm{GW}$ is fixed. This is called a medium or M-scenario for wind. In this paper we will not discuss the optimization of wind deployment, but a certain amount of wind capacity is needed to get a realistic estimation of the result of PV/battery deployment. In Belgium, an onshore capacity of I.5 GW and offshore of $0.7 \mathrm{GW}$ is present (in 20I6). The Medium wind scenario is assumed to be the most probable one. It is in line with other outlook reports [33];[34];[35];[36]. This M scenario may be fully deployed by $2025-2030$. The $M$ scenario is also the scenario, whereby no electricity from the wind generation need curtailment over a whole year, when no extra PV is installed.

Extra PV/battery installations are expected to increase the share of renewable energy in the electricity consumption. Therefore, the battery capacity (in kWh per kWp of PV capacity) was varied to quantify the impact of battery sizing on the share of renewable electricity consumed, for different levels of PV deployment ranging from 20 to $60 \mathrm{GWP}$, and for a 
fixed wind M-scenario. By changing the battery size between 0 and $2 \mathrm{kWh} / \mathrm{kWp}$, the share of renewable energy used for consumption is simulated and shown in Fig. 8 left. The fraction of curtailed renewable energy is shown in Fig. 8 right. The power injection limit for each deployment of PV is taken from Table I, the night injection limit to unload the battery from Table II.

A main insight is that higher battery capacities are appropriate for higher levels of PV deployment, but with an upper limit. If $20 \mathrm{GW}$ PV is combined with the Medium wind scenario (Fig. 8 left), the battery storage brings a relative minor increase of the renewable penetration. Only a 5\% extra renewable energy is used, when a net battery capacity of 2 $\mathrm{kWh} / \mathrm{kWp}$ is added. The difference between 0 and $2 \mathrm{kWh} / \mathrm{kWp}$ is increasing from $10 \%$ for the $30 \mathrm{GWP}$ case to $15 \%$ for $60 \mathrm{GWP}$. The numbers show the benefit of increasing battery storage capacity with increasing PV deployment. This is not surprising, as the more PV is installed, the lower the power injection limit becomes and the higher the rejected power. By storing a larger portion, this part of the PV generated electricity is injected during night time. This effect applies clearly between 0.5 and $1.5 \mathrm{kWh} / \mathrm{kWP}$. The more PV is installed, the higher renewable fraction is used with a higher battery capacity. This can intuitively be derived by the increase in the slope of the curves for increasing installed PV capacity. However, when we increase the battery capacity from 1.5 to $2 \mathrm{kWh} / \mathrm{kWp}$, the increase of the fraction of used renewable energy is flattening to I\% or less for all PV power capacities, as only during a limited amount of days, the battery is loaded above $1.5 \mathrm{kWh} / \mathrm{kWp}$ and at the same time, during night time, the injection level of $0.18 \mathrm{~kW} / \mathrm{kWp}$ (i.e. for the 2 $\mathrm{kWh} / \mathrm{kWp}$ ) competes with some wind electricity generated at that time. Moreover, the days the battery is loaded above $1.5 \mathrm{kWh} / \mathrm{kWp}$ is mostly during summer time, when the electricity consumption is lowest. In Belgium, the total electricity consumption during night time decreases in summer and during the Christmas holidays to about 6-8 GW [24].

From Fig. 6 an optimal battery capacity is derived as function of increasing PV deployment, i.e. the highest increase in the share of consumed renewable energy for a minimum increase of battery capacity. It is assumed that the battery remains, even in future, relatively expensive compared to PV and wind generation systems. The optimal PV/battery system combination for the highest increase in the share of consumed renewable energy is summarized in Table III. Up to $20 \mathrm{GWp}$, adding $2 \mathrm{kWh} / \mathrm{kWp}$ battery capacity ( $40 \mathrm{GWh}$ of batteries in total) results in less than $5 \%$ increase (Fig. 6 left). If $30 \mathrm{GWP}$ is deployed (I0 GWP extra PV on top of the $20 \mathrm{GWP}$ ) and adding $0.5 \mathrm{kWh} / \mathrm{kWP}$ battery capacity (I5 GWh of batteries in total), the share of consumed renewable energy increases with 6\% (Fig. 6 left). Even with future price evolutions, the cost for $10 \mathrm{GWP} P V$ installations combined with $15 \mathrm{GWh}$ of batteries is expected to be lower than $40 \mathrm{GWh}$ of batteries and therefore a more attractive scenario. But, with the model, other PV/battery combinations can easily be simulated and based on different price evolutions, other optimal PV/combinations could be derived.

By gradually increasing the battery capacity with increasing PV deployment (adding 0.5 $\mathrm{kWh} / \mathrm{kWp}$ per $10 \mathrm{GW}$ additional deployment (Table III), one can assure that the share of consumed renewable energy is increasing steadily between 7 and $9 \%$. Above $50 \mathrm{GW}$, increasing to $60 \mathrm{GWp}$, results in less than $5 \%$ extra renewable energy used, independent of extra battery capacity (see Table III). At such a high level of PV deployment, on a regular basis, all injected wind and PV energy is above the direct consumption and therefore more and more curtailed. When increasing the battery capacity for $60 \mathrm{GWp}$ from 1.5 to 2 $\mathrm{kWh} / \mathrm{kWp}$ it even reduces the renewable energy fraction, because a $2 \mathrm{kWh} / \mathrm{kWp}$ battery 
capacity competes during the night injection too often with wind generation, due to a lower electricity consumption during the night.

\section{Share of renewable energy for different grid injection limits}

Although with this combination of M-wind scenario and the optimal deployment of the PV/battery combination a large share of the electricity consumption can become renewable, still more than $20 \%$ of the annually generated renewable energy must be rejected, due to the power injection limit. As a final optimization, the power injection limit is reconsidered as function of PV power deployment to keep the share of consumed renewable energy as high as possible and minimizing the fraction of curtailed renewable energy. As a reminder, demand side management is still not considered here and only the algorithm of the power injection limit is varied. Until now, the power injection limit of the PV system was chosen, assuming all PV systems were mainly residential and therefore injecting all generated PV power into the LV grid. Part of the PV electric energy is also generated by PV power plants or PV on large buildings, as railway stations or sound barriers of highways. These types of large PV systems inject in the medium or high voltage grid. The MV and HV grids have not the same limitation for low injection capacity as the LV grid and can cope with higher power injection levels to transmit them over a larger area, without power congestion. If part of the PV system is also injected into the medium or high voltage grid, the power injection limit can be raised as long as the low voltage part does not exceed in total the $6 \mathrm{GW}$ limit, which is used as a limit in this study.

For the optimal PV/battery combinations (Table III) and with the medium wind scenario, the share of consumed renewable energy as function of the power injection limit of the PV installations during day time is recalculated. The power injection limit of the batteries during the night is kept the same as in Table II. The results are shown in Fig. 7. For each $\mathrm{PV} /$ battery combination, a maximum of consumed renewable energy share is obtained for a specific power injection limit. A main conclusion is that the larger the PV deployment is, the lower is the optimal power injection limit. For $20 \mathrm{GWP}$ of PV total installed power, an injection limit of 0.4 to 0.5 would be more optimal than the $0.3 \mathrm{~kW} / \mathrm{kWp}$, needed when only residential PV systems are installed (Table I). For $50 \mathrm{GWP}$ the optimum is around 0.2 $\mathrm{kW} / \mathrm{kW}$. A further optimization of power injection limit combined with a differentiation of the PV power injection on the LV grid and the MV and HV grids improves the penetration of renewable electricity in the grid.

\section{Deployment scenario for renewable energy in Belgium}

Based on the results in previous section, a scenario is proposed for the deployment of wind and $\mathrm{PV}$, in combination with a gradual reduction of the PV injection limit and a gradual buildup of battery capacity, to increase in an effective and realistic way the share of consumed renewable energy in Belgium. The scenario illustrates that it is possible to enable a rapidly increasing share of consumed renewable energy in the initial stages of deployment by applying the power injection limit only, while battery capacity needs to be added in later stages when renewables become the dominant electricity source.

In Table IV, an overview is shown in phases to realize these renewable energy capacities. It starts the situation in 2016 and proposes an evolution in phases towards a certain capacity of wind and PV. To realize this capacity, the parameters for injection levels (night and day) and battery capacity are optimized based on the results of previous section and listed in 
Table IV. The final share of renewable energy consumed and fraction of renewable energy curtailed (Table IV) is therefore slightly different compared to the data in the results section. The power injection limits during the day in Table IV are taken from the maxima in Fig. 7 and differ from the proposal in Table I. The consequence of the slightly higher power injection limits, is the reduced amount of PV installations that can be allowed on the Belgian LV grid. This optimized proposal gives also a guidance to the maximum deployable PV installations connected to the LV grid and the required PV installations to be connected to the MV and HV grid to reach these renewable energy fraction as listed in Table IV. For the sake of simplicity, the same power injection limit and battery storage capacity for the PV installations on the LV, MV and HV grids are used. A further study may refine the grid injection power limit for the LV, MV and HV grids as well as optimizing the storage capacities for each type of grid. Therefore, the share of renewable energy consumed in Table IV, is the worst-case result with the use of simple algorithms for the day and night power grid injection limits and the battery storage capacities connected to the PV installations. More detailed control, combined with smarter algorithms may increase the renewable energy share further and reduce the curtailed renewable energy fraction. The phases in Table IV may be considered as milestones in a project plan, whereby the deployment of specific wind and PV capacities, imply specific settings of the different model parameters (PV capacity on LV vs. MV/HV grid, day and night injection levels and battery capacity) to maximize the share of renewable energy and minimize the curtailed renewable energy. Although a realistic time line may be suggested to achieve these milestones, policy makers may still consider different options for deployment. Results of these choices can be calculated using this model. In the Belgian plan for renewables, a considerable amount of wind deployment is already foreseen [26]; [29] and phase I and 2 is increasing the wind capacities up to the medium wind scenario, considered as a realistic level. PV deployment is complementing this wind deployment in phase I and 2 up to $20 \mathrm{GWp}$. To realize this PV deployment, no main grid upgrades nor main battery installations would be needed to achieve an almost $50 \%$ share of consumed renewable energy. The timing of phase 2 could be between 2025 and 2030, dependent on policy measures. For wind deployment, it means to install a yearly capacity from 2018 on, between 250 and $500 \mathrm{MW}$ for offshore and for onshore wind turbines. For PV deployment, some plans [26];[29] predict a more conservative growth, but values up to $20 \mathrm{GWp}$ are in line with the outlooks. The scope of Table IV is to calculate how the share of consumed renewable energy would increase beyond phase 2, if for each phase, an additional $10 \mathrm{GWP}$ of $\mathrm{PV}$ and $0.5 \mathrm{kWh} / \mathrm{kWp}$ of batteries are installed.

At phase 2, the share of consumed renewable energy is already near $50 \%$ and less than $10 \%$ of the renewable energy must be curtailed. In this case the optimal injection limit is 0.4 $\mathrm{kW} / \mathrm{kW}$ and therefore the PV installations connected to the LV grid should be limited to I5 GW . Above $20 \mathrm{GWP}$ PV installations, batteries are needed to avoid too much curtailment of the generated PV electricity. The optimal combination of PV/battery capacities are the same as in Table III. By increasing the PV capacity to $50 \mathrm{GWp}$, with gradually lowering the injection limit and gradually increasing the battery capacity per $\mathrm{kWp}$ installed $\mathrm{PV}$, the share of consumed renewable energy increases about $7 \%$ per ' $10 \mathrm{GW} / 0.5$ $\mathrm{kWh} / \mathrm{kWp}$ ' added 'PV/battery' installations. At $50 \mathrm{GWP}$ (phase 5), the share of consumed renewable energy is almost $70 \%$ and the fraction of curtailed renewable energy becomes $20 \%$ at that point. The final scenario of $60 \mathrm{GWP}$ (light grey marked) is added to the table to show that the PV capacity from 50 to $60 \mathrm{GWP}$ (using these optimal injection level parameters) only results in an extra renewable energy fraction of $3 \%$, which is probably no longer cost-effective anymore. 


\section{Discussion}

\section{Cost savings of this approach}

The impact on the cost for the total electricity system in Belgium of this proposal is not quantified in this study. It also depends on the future regulatory framework on energy. It needs a more careful study, taking also potential new business models into account and the impact on the local economy of the introduction of these new technologies. Therefore, only qualitative cost estimations of this proposal are given to clarify some main cost advantages of this proposal:

- By using a grid power injection limit on the LV grid, no upgrades are needed in the existing grid in Belgium, except a few local ones. The cost of grid upgrades, when a massive amount of PV power is introduced at sunny noon's, is a typical cost argument against the deployment of PV. This proposal solves this issue.

- The direct use of generated renewable power is in this proposal maximized. This is the most cost-effective way to use renewable power.

- When curtailment becomes too high, batteries are used to store the energy up to the point they are fully charged. In this proposal, the suggested time to install a large base of home batteries is about 10 years from now. By then, it is expected battery prices are dropped drastically, making it more cost-effective compared to immediate installation now. But, it is difficult to predict the exact price decline in the future. In retrospective, $\mathrm{PV}$ prices declined over the past 10 years much more than the most optimistic prediction in 2008. If batteries in the next 10 years would undergo the same price decline is impossible to predict.

- The algorithms used for the grid power injection limitation and charging/discharging of the batteries are simple and equal for every prosumer on the grid. For all prosumers, it is an equal level playing field.

In summary, this proposal is a no-regret scenario for the deployment of renewable energy resources. At the start of the proposed scenario, the generated power is used for direct consumption. This is by far the most cost-effective use of renewable resources and at this moment already competitive with non-renewable power generation. By the time batteries are needed in the system to increase the renewable share, the exact deployment of batteries can be reconsidered, especially when battery prices decline rapidly.

\section{Impact of demand side response and end-use sector coupling}

The calculation of the fraction of curtailed renewable energy was based on the present consumption profiles of the years 2014, 2015 and 2016. The potential of demand side response is key to reduce this curtailment, both through industrial and residential applications. PV is curtailed by limiting the PV and battery injection in the grid and wind is curtailed, when the renewable energy is above the consumption for that hour. Such a curtailment must be considered as the worst-case scenario. In Table V, the data of Table IV are further elaborated and the fraction of curtailed renewable energy is split between the PV curtailed part and the wind curtailed part. The wind part is always injected on the HV or MV grid. Based on both curtailed parts, combined with the division in Table IV of PV capacity on the LV and the MV/HV grid, the fraction of curtailed renewable energy is calculated in Table $V$ for the potential to be injected in the LV grid or in the MV/HV grid. 
For the phase 5 (50 GWP PV and wind M-scenario), I2\% of the total curtailed renewable energy (PV and wind capacities) is connected to the MV/HV grid. There are almost no constraints for this renewable energy to be distributed over the MV and HV grid. This curtailed electricity can be injected and consumed elsewhere, when the appropriate demand response arrangements are present. E.g., this energy could be used for thermal processes in the industry (connected to HV and MV grids), which now are mainly generated by fossil fuels (typically natural gas based). The additional investment of electrical heaters inside a gas heater is very cost-effective, when this surplus renewable energy would be offered at a reasonable price (i.e. below the gas price). The advantage is that industrial thermal processes, which now are mainly using fossil fuels, are then partially replaced by this (otherwise curtailed) renewable energy and reduces directly the $\mathrm{CO}_{2}$ output. In Belgium, for the scenario with $50 \mathrm{GWP}$ of PV, about 7 TWh (I2\% of 60 TWh annually generated renewable energy) of industrial thermal processing energy from fossil fuels may be replaced by the this curtailed renewable energy. In addition, district heating may be used to store excess electricity as heat [37]. In Belgium, district heating is currently deployed only at small scale. Nonetheless, there is ample of policy attention for developing district heating in the transition towards a low carbon energy system [38], which would add another potential usage for curtailed electricity.

The trend of electrification in domestic heating and mobility may offer opportunities for reducing the level of curtailed energy from $\mathrm{PV}$ via residential demand response. For the $7.5 \%$ renewable energy curtailed in the $50 \mathrm{GWP}$ scenario at the PV part connected to the LV grid, the surplus PV generated electricity could be used for heating sanitary water (with buffer tank) or for heat pumps used for cooling in summer time (the main part of this curtailed energy is produced in summer time).

For reference, in Belgium, about 10 TWh per year [39] is used for domestic (residential and tertiary) sanitary water heating (total of electrical and fossil fuel heating), while the $7.5 \%$ curtailed PV would represent 4.5 TWh/year in the $50 \mathrm{GW}$ p scenario.

Another possibility would be to use this curtailed part at home for charging the car battery in the future. According to [7], a significant increase in power demand can be expected in the transport sector adding up to some $2.5 \mathrm{TWh}$ annually by 2030 , roughly equivalent to 650,000 to 700,000 electrical cars in Belgium by 2030 . As a conclusion, it is not certain, that with demand response participation, the full 7.5\% curtailed PV electricity is possible to be used. However even if a small portion is still curtailed, it would still be a very cost-effective way to increase the share of renewables in the Belgian energy mix.

Potential of dynamic control of the grid injection limits and flexible use of the battery as well as import/export with neighboring countries

In this study, a very simple and static algorithm for the day and night power grid injection limit throughout the whole year is proposed. This is leading to a relative high curtailment to protect the LV gird as proposed in this study. In further studies, the impact of a dynamic control (e.g. by the grid operator) on this grid injection limit for the PV installations during day time and for the batteries at any time, could be investigated. This could increase further the share of renewable energy consumed and reducing the fraction of the curtailed renewable energy. Other measures to reduce the curtailed part could be to investigate the potential of import and export with neighboring countries of Belgium, because the Belgian $\mathrm{HV}$ grid is well connected with its neighbors. 


\section{$\underline{\text { Relevance of social acceptance }}$}

The shift from self-consumption to direct usage presents an interesting challenge for social acceptance and engagement [40]; [4I]. Whereas the concept of self-consumption appeals to notions of independence and autonomy that are valued by users [40], the concept of direct usage is rather based on the rationale of system efficiency, which may be a more abstract notion for users. Moreover, the curtailment of energy - which is rational from the perspective of system efficiency - may be consider un-rational from the user perspective. Therefore, societal perspectives on the concept of direct usage as discussed in this paper need to be further assessed, and user engagement will be required to enable full scale deployment.

\section{Conclusions}

An overall concept for increasing the penetration of renewable electricity production in the Belgium grid using the existing consumption profiles is proposed, by maximizing the direct use of generated renewable electric power. The potential problem of grid overload on the Low Voltage grid at sunny midday periods is tackled in this study. Balancing the maximum available wind capacity, with an optimized PV capacity (using a simple algorithm for a grid injection limit during day time) and optimized battery capacity (using a simple algorithm for a grid injection limit during night time) provides the best results. It can obtain almost $70 \%$ of renewable electricity consumption with limited electrical storage deployment and no or minimal modifications to the low voltage grid. The model would also allow to estimate the potential in the neighboring countries of Belgium to increase the renewable energy generation with existing grid infrastructure. 


\section{Appendix A: Validation of the results for different years}

To validate the results and conclusions of Table IV, which is using the numbers from 2015 for the simulation, simulations with the input parameters of Table IV were carried out for the years 2014 and 2016 for the availability factors of PV, onshore and offshore wind and the electricity consumption on an hourly basis. In Fig. AI (left), the share of consumed renewable energy for all the scenario's is varying less than $2 \%$ between the 3 years (phase 5 numbers are: $2014=68 \% ; 2015=69 \% ; 2016=67 \%$ ). The fraction of curtailed renewable energy (Fig. Al right) is varying less than $5 \%$ between the 3 years (phase 5 numbers are: $2014=16 \% ; 2015=20 \% ; 2016=18 \%)$.

\section{Acknowledgments}

This project receives the support of the European Union, the European Regional Development Fund ERDF, Flanders Innovation \& Entrepreneurship and Province of Limburg. The authors like to acknowledge the EFRO-SALK project 'Towards a Sustainable Energy Supply in Cities', for their support. 


\section{References}

[I] United Nations Climate Change, http://unfccc.int/paris_agreement/items/9485.php (accessed 2 January 2018).

[2] Christian Breyer, Dmitrii Bogdanov, Arman Aghahossein, Ashish Gulagi, Michael Child, Ayobami Solomon Oyewo, Javier Farfan, Kristina Sadovskaia, Pasi Vainikka. Solar

photovoltaics demand for the global energy transition in the power sector, Prog. In PhotoV. (2017), https://doi.org/10.1002/pip.2950

[3] Christian Breyer, Dmitrii Bogdanov, Ashish Gulagi, Arman Aghahosseini, Larissa S.N.S. Barbosa, Otto Koskinen, Maulidi Barasa, Upeksha Caldera,

Svetlana Afanasyeva, Michael Child, Javier Farfan and Pasi Vainikka. On the role of solar photovoltaics in global energy transition scenarios. Prog. Photovolt: Res. Appl. (2017), DOI: 10.1002/pip.2885.

[4] International Energy Agency (IEA), Report on 'World Energy Outlook 2017' (2017), . https://www.iea.org/weo20I7l, (accessed 2 january 2018).

[5] REN2I Report, http://www.ren2I.net/status-of-renewables/global-status-report/

[6] IRENA Report, http://www.irena.org/publications/2018/Apr/Global-Energy-Transition-ARoadmap-to-2050

[7] Frank Meinke-Hubeny, Larissa P. N. de Oliveira, Jan Duerinck, Pieter Lodewijks, Ronnie Belmans. "Energy Transition in Belgium - Choices and Costs",

http://www.energyville.be/nieuwsbericht/energy-transition-belgium-choices-and-costs ;

(28APR, 20I7) [accessed 20 dec 20I7].

[8] Vision document for the Belgian federal energy pact (in Dutch):

https://emis.vito.be/sites/emis.vito.be/files/articles/3331/2018/Visienota_-

BE_Interfederaal_Energiepact_209.pdf

[9] Kris Kessels, Carolien Kraan, Ludwig Karg, Simone Maggiore, Pieter Valkering and Erik Laes, Fostering Residential Demand Response through Dynamic Pricing Schemes: A Behavioural Review of Smart Grid Pilots in Europe (2016). Sustainability 2016, 8(9), 929; doi: $10.3390 /$ su8090929.

[10] Siano, P., Demand response and smart grids - A survey (20I4). Renewable and Sustainable Energy Reviews, Vol. 30, February 2014, Pp. 46I-478.

[I I] O'Connell, N., Pinson, P., Madsen, H., O'Malley, M. (20I4). Benefits and challenges of electrical demand response: A critical review. Renewable and Sustainable Energy Reviews 39, 686-699.

[12] The report LINEAR: "Demand Response for Families" (www.linear-smartgrid.be), http://www.linear-smartgrid.be/sites/default/files/boekje_linear_okt_2014 boekje_web.pdf; (20I4) [accessed 20 dec 20I7].

[13] Nicholas Good, Keith A. Ellis, Pierluigi Mancarella, Review and classification of barriers and enablers of demand response in the smart grid (2017). Renewable and Sustainable Energy Reviews, Vol. 72, May 2017, pp. 57-72.

[14] Agora Energiewende (https://www.agora-energiewende.de/en), publications:

'Renewables versus fossil fuels - comparing the costs of electricity systems' (july 2017). https://www.agora-energiewende.de/en/topics/-agothem-

/Produkt/produkt/353/Erneuerbare+vs.+fossile+Stromsysteme\%3A+ein+Kostenvergleich/ (accessed 02 january 2018).

[15] Andreas Zucker, Timothée Hinchliffe, Optimum sizing of PV-attached electricity storage according to power market signals - A case study for Germany and Italy (2014), Applied Energy, Vol. I27, pp. I4I-I55.

[16] Chun Sing Lai, Malcolm D. McCulloch, Levelized cost of electricity for solar photovoltaic and electrical energy storage (20I7), Applied Energy, Vol. I90, pp. I9I-203. 
[17] Björn Nykvist and Måns Nilsson, Rapidly falling costs of battery packs for electric vehicles (2015), Nature Climate Change 5, 329-332 (2015).

[18] Kittner, Noah; Lill, Felix; Kammen, Daniel M., Energy storage deployment and innovation for the clean energy transition, Nature Energy, Volume 2, Issue 9, pp. 17125 (2017), DOI: 10.1038/nenergy.2017.125.

[19] O. Schmidt, A. Hawkes, A. Gambhir \& I. Staffell, The future cost of electrical energy storage based on experience rates, Nature Energy volume 2, Article number: I7I I0 (20I7), DOI: 10.1038/nenergy.2017.110.

[20] Matthias Deutsch and Patrick Graichen. 'What if... there were a nationwide rollout of PV battery systems?' (Oct 20I5). Agora Energiewende (https://www.agoraenergiewende.de/en), https://www.agora-energiewende.de/fileadmin/Projekte/20I5/PVSpeicher-Rollout/Agora_Speicherdurchbruch_2015-10-08_web_EN.pdf (accessed I5 november 2017).

[2I] Guilherme de Oliveira e Silva and Patrick Hendrick. Photovoltaic self-sufficiency of Belgian households using lithium-ion batteries, and its impact on the grid (2017). Applied Energy 195 (2017) 786-799.

[22] Fares and Webber, The impacts of storing solar energy in the home to reduce reliance on the utility (20I7). NATURE ENERGY 2, I700I (20I7), DOI: 10.1038/nenergy.20I7.I

[23] Green, R. and Staffell, I., "Prosumage" and the British electricity market (2017).

Economics of Energy and Environmental Policy 6 (I), 33-49.

[24] Elia, Belgium's electricity transmission system operator, grid data,

http://www.elia.be/en/grid-data/ (assessed 2 january 2018).

[25] Communication in Commission of Flemish Parliament on 8 november 2017:

'Hoorzitting over het ontwerp van ondernemingsplan en ontwerp van begroting van de

Vlaamse Regulator van de Elektriciteits- en Gasmarkt (VREG)'

[26] Benjamin Matthiss, Dirk Stellbogen, Maria Eberspächer and Jann Binder. Curtailed Energy of PV Systems - Dependency on Grid Loading Limit, Orientation and Local Energy Demand. Proc. Of 3 I st EUPVSEConfernce, 23II-23| 4 (2016).

[27] Eberhard Waffenschmidt. Dimensioning of decentralized photovoltaic storages with limited feed-in power and their impact on the distribution grid. Energy Procedia 46 ( 2014 ) $88-97$

[28] Report on: Cost-effective integration of photovoltaics in existing distribution grids: results and recommendations. http://www.metapv.eu/FinalReport; (20I5) [accessed $20 \mathrm{dec}$ 20I7].

[29] Yannick Riesen, Christophe Ballif, Nicolas Wyrsch, Control algorithm for a residential photovoltaic system with storage (2017), Applied Energy Vol. 202, Pp. 78-87

[30] Yancheng Zhang and Chao-Yang Wang,Cycle-Life Characterization of Automotive Lithium-Ion Batteries with LiNiO2 Cathode, J. Electrochem. Soc., I 56 issue 7I A527-A535 (2009).

[3I] Wessells, C. D. et al. Copper hexacyanoferrate battery electrodes with long cycle life and high power. Nat. Commun. 2:550, DOI: 10.1038/ncomms I563 (20I I).

[32] M. Agostini, S. Brutti, M. A. Navarra, S. Panero, P. Reale, A. Matic \& B. Scrosati, A highpower and fast charging Li-ion battery with outstanding cycle-life, Scientific Reports, volume 7, Article number: II 04 (2017).

[33] Federaal planbureau (http://www.plan.be). Report on: Het Belgische energiesysteem in 2050: Waar naartoe? - Beschrijving van een Referentiescenario voor België (Oct 2014).

(Dutch and French version)

http://www.plan.be/publications/publication-I388-en-

het+belgische+energiesysteem+in+2050+waar+naartoe+beschrijving+van+een+referentiesc enario+voor+belgie (20l4) [accessed $20 \mathrm{dec} 2017$ ]. 
[34] Esch, L.V., Meynaerts, E., Vermeiren, K., Uljee, I., Janssen, L., Guisson, R., Engelen, G., Robeyn, H.H.e.N. “Hernieuwbare EnergieAtlas Vlaamse gemeenten”, VITO, TerraEnergy, Belgium (2016).

[35] Infrax, Eandis, Elia. “Onthaalcapaciteit decentrale productie in Vlaanderen 20 I I-2020”, Studie, Belgium, p. I33 (20I2).

[36] Electricity scenarios for belgium towards 2050 - Elia's quantified study on the energy transition in 2030 and 2040 (http://www.elia.be), http://www.elia.be/ /media/files/Elia/AboutElia/Studies/2017III4_ELIA_4584_AdequacyScenario.pdf; (november 2017) [accessed 20 dec 2017].

[37] Lund, H., Østergaard, P. A., Connolly, D., \& Mathiesen, B. V. (20I7). Smart energy and smart energy systems. Energy. https://doi.org/10.1016/j.energy.2017.05.123

[38] The Flemish heat plan (in Dutch): https://www.energiesparen.be/warmteplan

[39] Odyssee-MURE project (http://www.odyssee-mure.eu/), Data for the year 20I3,

Belgian sanitary water heating (20l4). [accessed on I5/0I/20I8]

[40] Valkering, P., et al., How to engage end-users in smart energy behaviour? (20I4), EPJ Web of Conferences 79.

[4I] Ellabban, O. and H. Abu-Rub. Smart grid customers' acceptance and engagement: An overview (2016). Renewable and Sustainable Energy Reviews, 65: I 285-1298. 


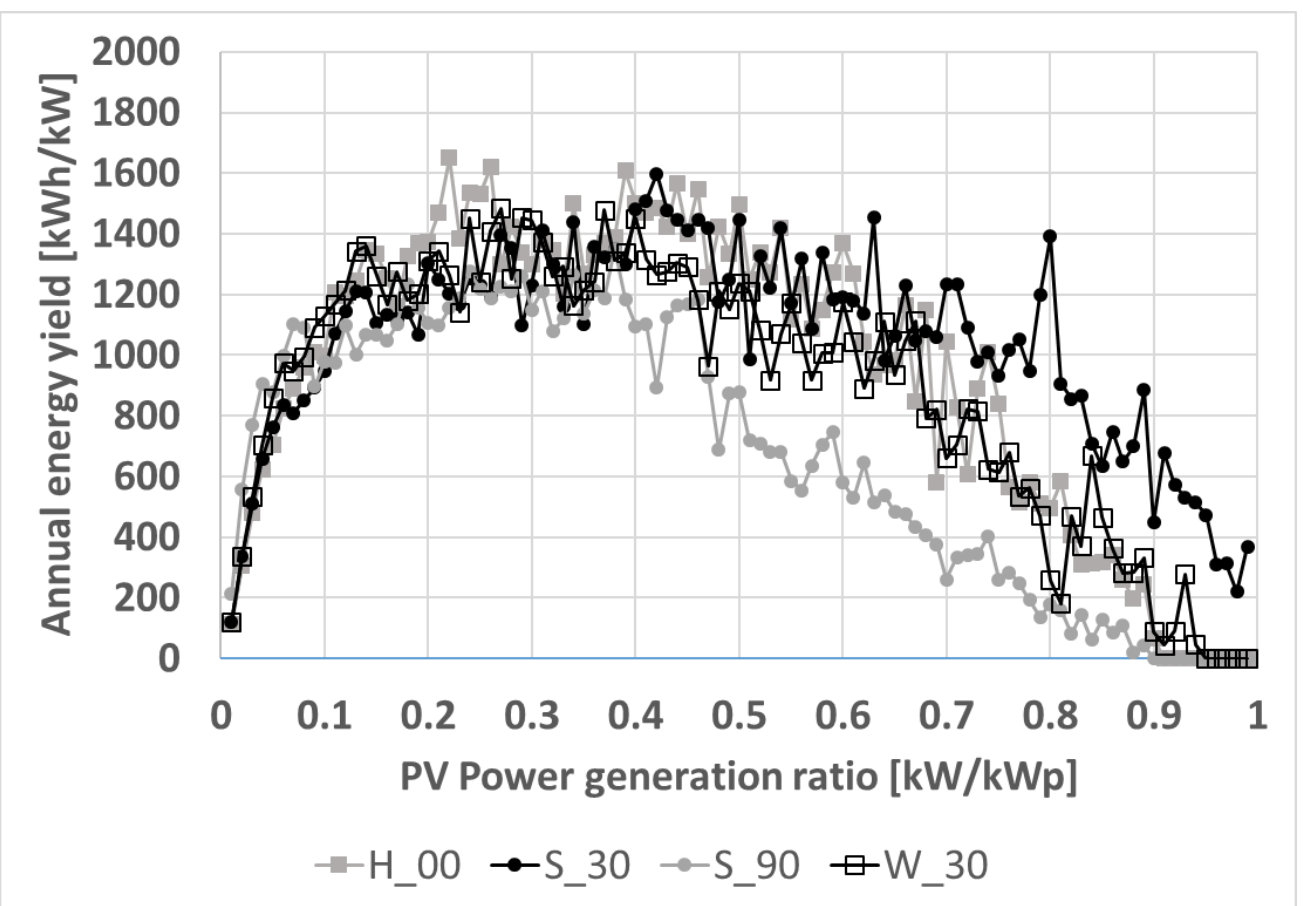

Fig. I: Normalized annual energy yield as a function of the generated power of a PV system of I $k W p$, installed under 4 different orientations (Horizontal $-0^{\circ}$ inclined, South-30 $0^{\circ}$ inclined, South $90^{\circ}$ inclined (vertical) and West $-30^{\circ}$ inclined). The annual energy yield (in kWh) is normalized to the power interval (in kW).
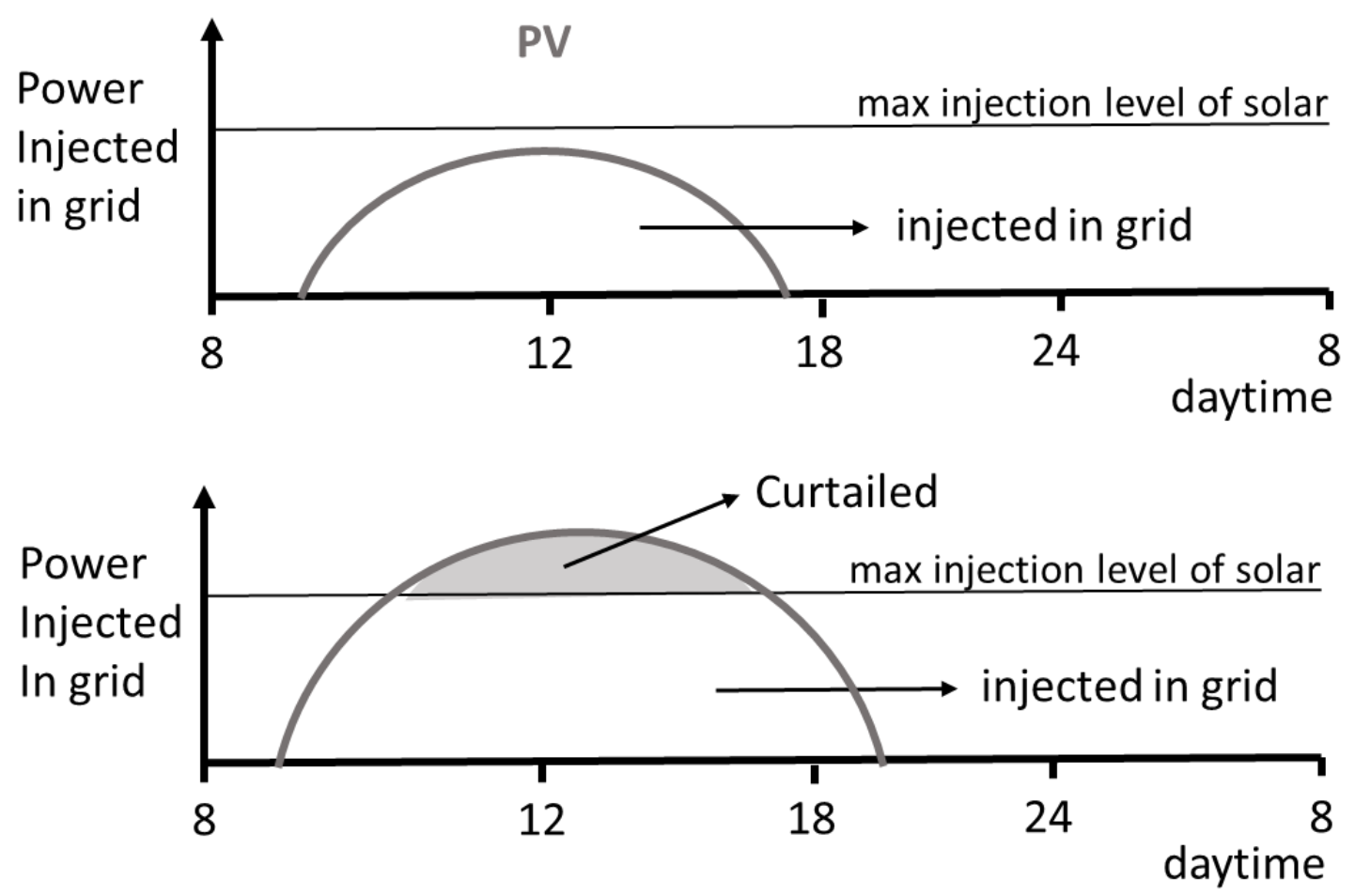

Fig. 2: Schematic illustration of the proposed power grid injection limitation algorithm from PV panels without battery. Top figure: the net PV production power is not reaching the maximum injection level. All PV generated power is injected into the grid. Bottom figure: the net PV power generated at power levels above the maximum injection level is not injected in the grid. 

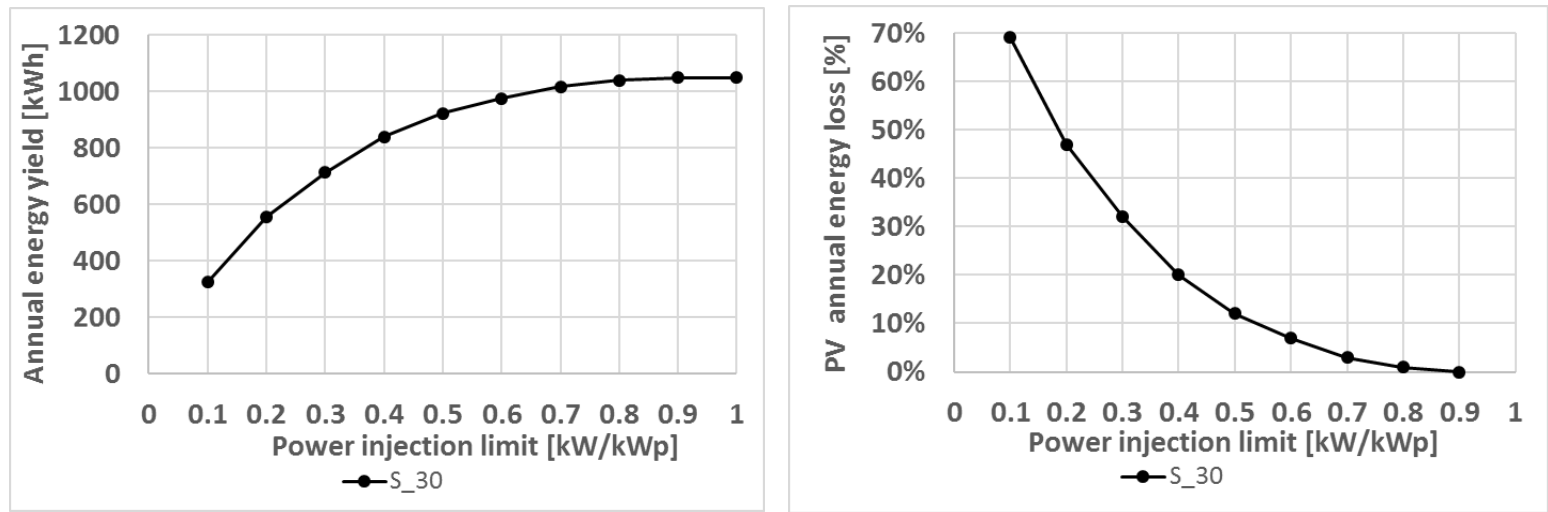

Fig. 3 (left): generated PV annual energy yield as function of the power grid injection limit for S-30 orientation.

Fig. 3 (right): PV annual energy yield loss (in \% of the total annual energy yield at I kW/kWp) as function of the power grid injection limit.
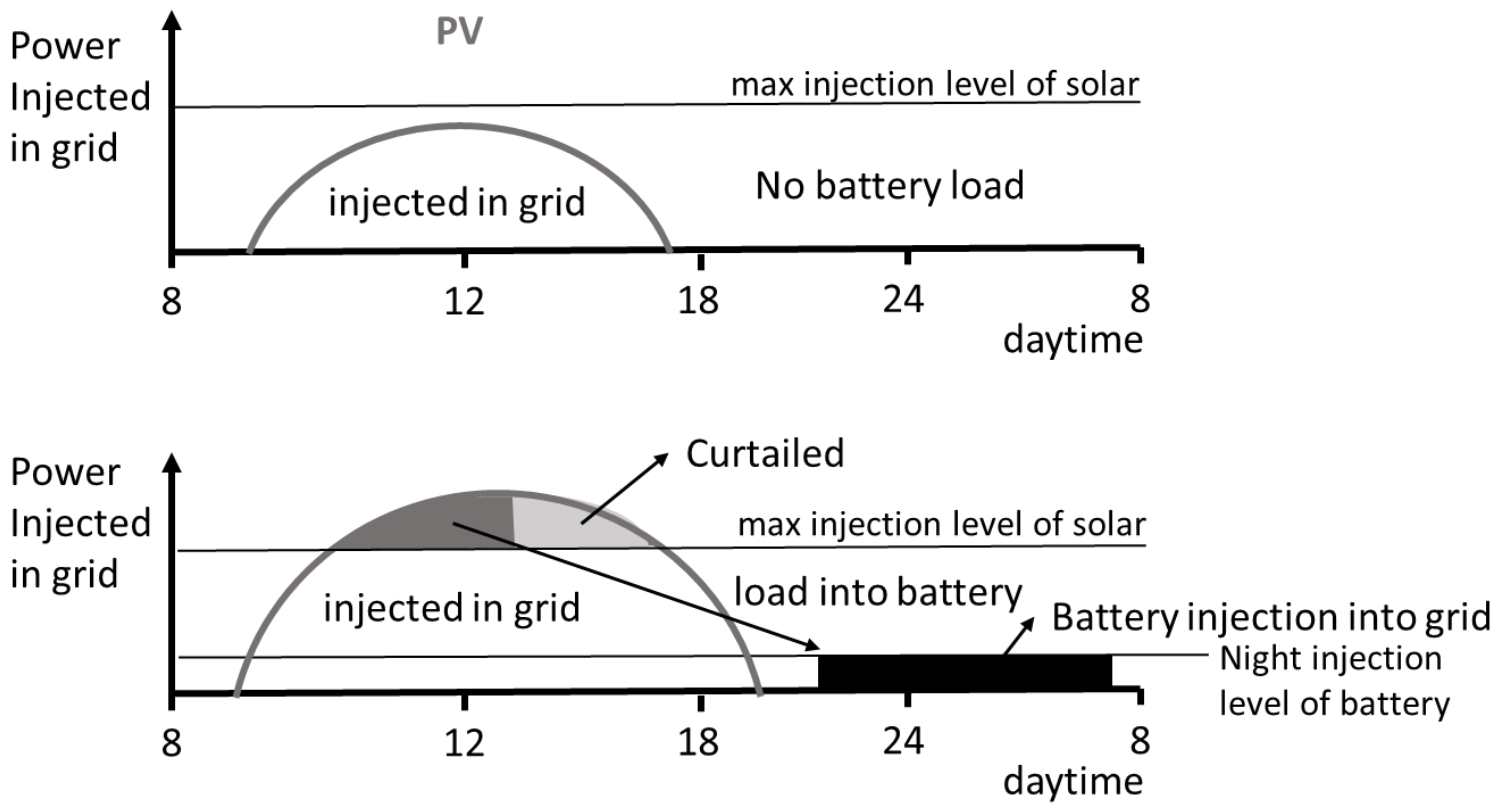

Fig. 4: Proposed injection algorithm for PV with battery. Top figure: when the solar panels deliver less than the maximum injection level, the battery is not loaded and all power is injected in the grid during day time. Bottom figure: The PV power produced above maximum injection level, is stored in the battery until its capacity is reached. The extra electric energy generated when the battery is full, is still rejected. At $2 \mathrm{I} h$ in the evening, the battery is discharged at constant power during maximum IOh and delivers to the grid at a lower night injection level. It ensures that during night time, the electricity injected by the battery can be consumed in a useful way. 


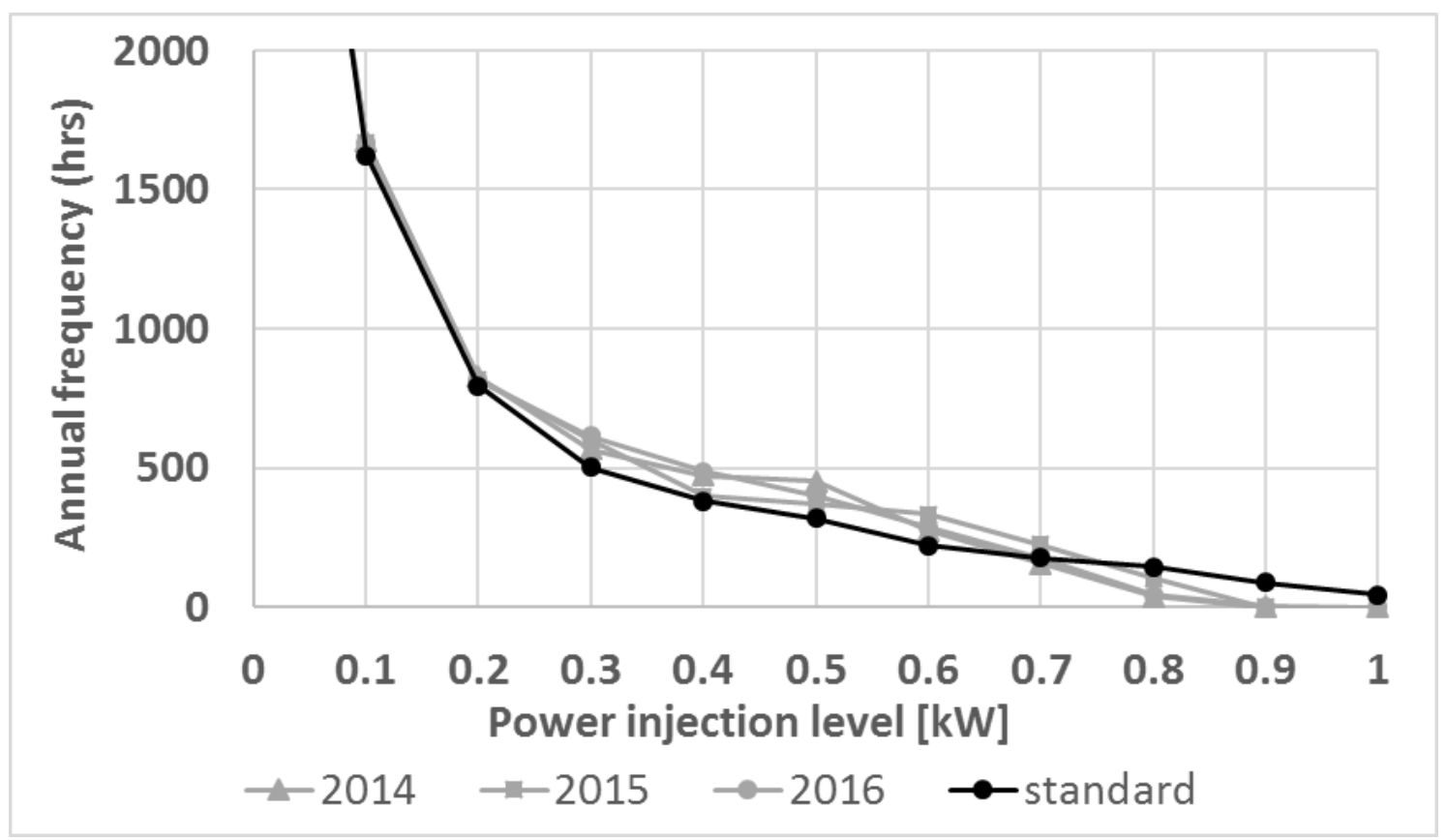

Fig. 5: Comparison between hourly injected PV power on the Belgian grid for real time values in 2014,2015 en 2016 and compared to the calculated hourly power production for a I kWp S-30 oriented PV system based on a standard sun irradiation year in Uccle, Belgium.
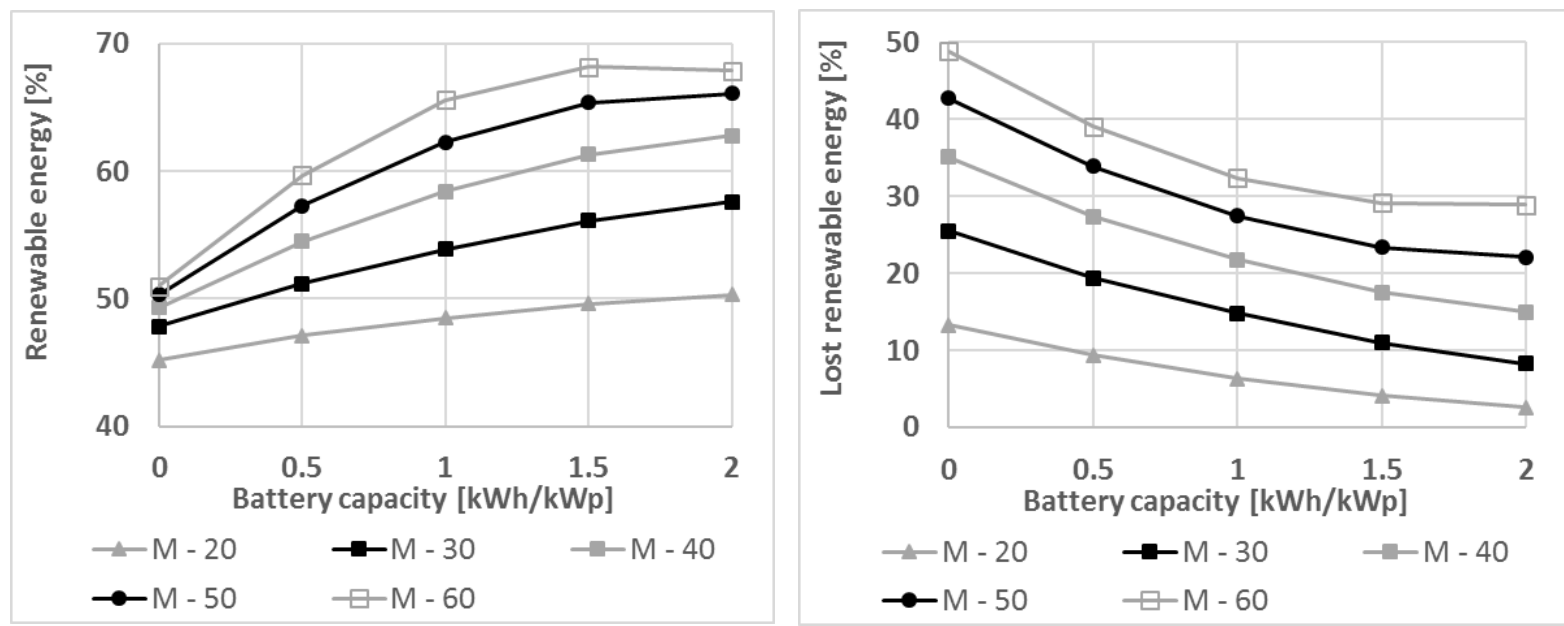

Fig. 6: (left) Share of the renewable energy injected in the Belgian grid as function of PV/battery combinations for the Medium-wind scenario. (right) Fraction of the curtailed renewable energy as function of PV/battery combinations for the Medium-wind scenario. 


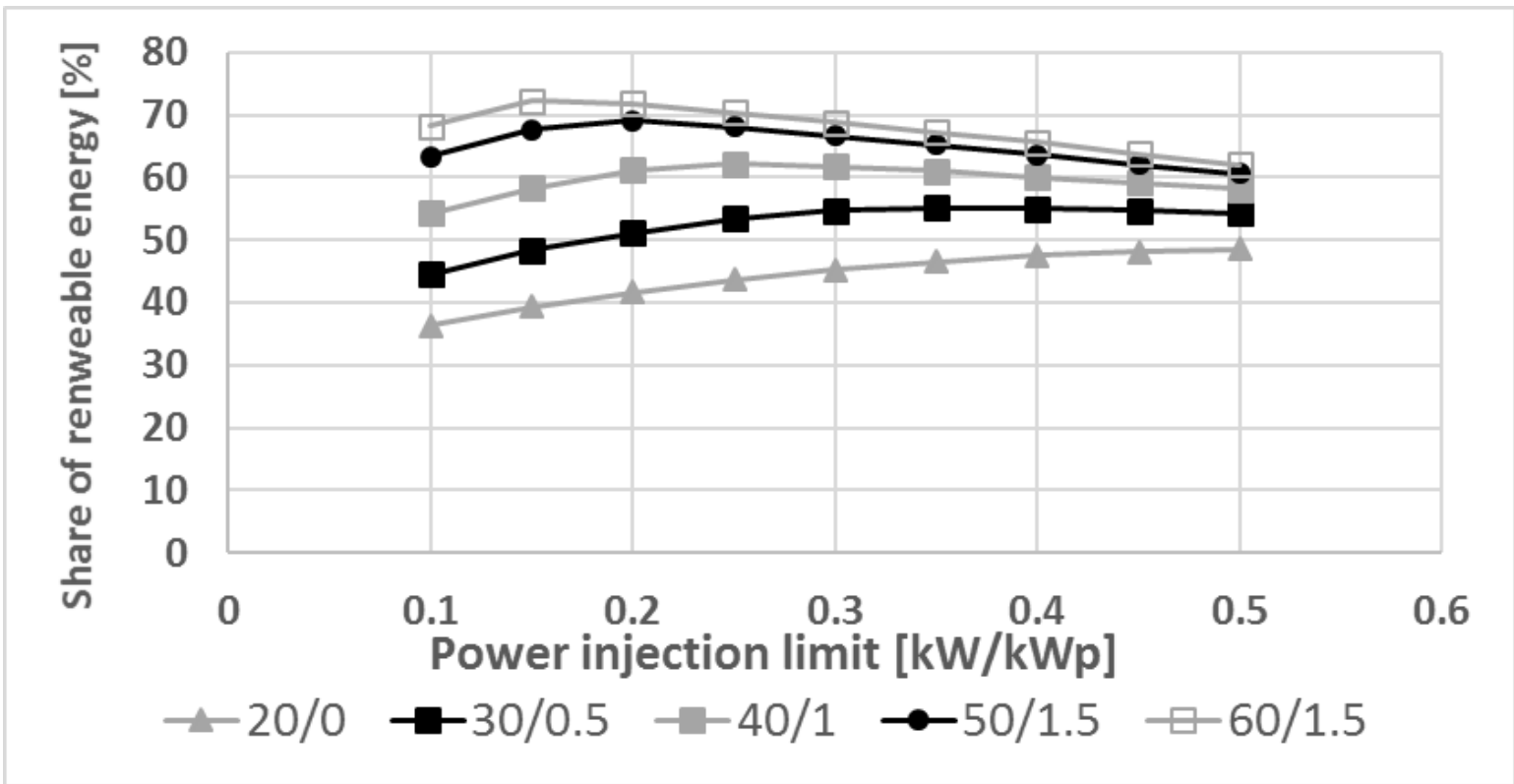

Fig. 7: Share of consumed renewable energy injected into the grid as function of power injection limit for 5 different PV/battery combination listed in Table III.
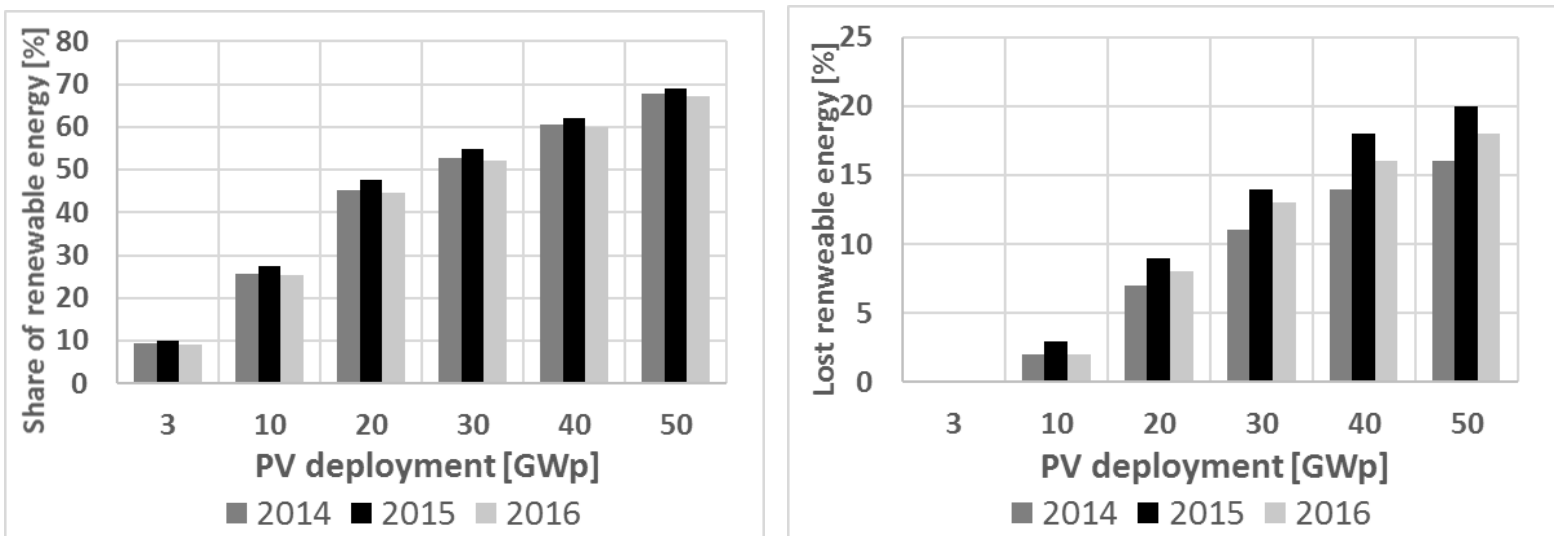

Fig. Al: (left) Share of renewable energy consumed and; (right) fraction of curtailed renewable energy as function of the PV deployment with input parameters of Table IV. The modeling is based on the availability factors of $P V$, onshore wind, offshore wind and electricity consumption on an hourly basis for the years 2014, 2015 and 2016. 
Table I: Maximum grid injection limit as function of the PV system deployment to allow a maximum of $6 \mathrm{GW}$ injection from all PV systems.

\begin{tabular}{|l|c|c|c|c|c|c|c|c|}
\hline Total GWP solar installed & 5 & 10 & 15 & 20 & 30 & 40 & 50 & 60 \\
max injection level of solar (kW/kWP) & 1 & 0.5 & 0.4 & 0.3 & 0.2 & 0.15 & 0.12 & 0.10 \\
maximum PV injected in LV grid (GW) & 5 & 5 & 6 & 6 & 6 & 6 & 6 & 6 \\
\hline Rejected electricity for ideal PV orientation & $0 \%$ & $12 \%$ & $20 \%$ & $32 \%$ & $47 \%$ & $58 \%$ & $65 \%$ & $69 \%$ \\
\hline
\end{tabular}

Table II: Night injection level (between 2Ih-7h) for different battery capacities on the PV system.

\begin{tabular}{|l|c|c|c|c|}
\hline Net battery capacity on PV system $(\mathrm{kWh} / \mathrm{kWP})$ & 0.5 & $\mathrm{I}$ & $\mathrm{I} .5$ & 2 \\
\hline Night injection level of battery $(\mathrm{kW} / \mathrm{kW})$ & 0.045 & 0.09 & 0.135 & 0.18 \\
\hline
\end{tabular}

Table III: Optimal PV/battery size combination for a $\mathrm{M}$ wind scenario to increase the share of consumed renewable energy in the grid and to reduce the fraction of curtailed renewable energy. For the $60 \mathrm{GWp}$ of PV capacity, a comparison is given for 1.5 and $2 \mathrm{kWh} / \mathrm{kWp}$ battery capacities, demonstrating that this extra battery capacity will not necessarily lead to a higher share of renewable energy consumed.

\begin{tabular}{|l|c|c|c|c|c|c|}
\hline Total GWP solar installed & 20 & 30 & 40 & 50 & 60 & 60 \\
Net battery capacity [in kWh/kWP] & 0 & 0.5 & $\mathrm{I}$ & 1.5 & 1.5 & 2 \\
\hline Share of renewable energy (SRE) [\%] & 45.2 & $5 \mathrm{I} .2$ & 58.4 & 67.7 & 72.3 & 72.0 \\
\hline Fraction of RE curtailed (FRC) [\%] & 13.3 & 20 & 23.1 & 23 & 27.6 & 27.9 \\
\hline
\end{tabular}

Table IV: Proposal for deployment of renewable energy on the Belgian electricity grid simulated for electrical consumption data and availability factors of wind and PV in the year 2015.

\begin{tabular}{|l|c|c|c|c|c|c|c|}
\hline Phase of deployment & 2016 & 1 & 2 & 3 & 4 & 5 & $\mathrm{n} / \mathrm{a}$ \\
\hline Wind capacity offshore [GW] & 0.7 & 2.2 & 4 & 4 & 4 & 4 & 4 \\
Wind capacity onshore [GW] & 1.5 & 3 & 5 & 5 & 5 & 5 & 5 \\
\hline Total PV capacity [GWP] & $\mathbf{3}$ & $\mathbf{1 0}$ & $\mathbf{2 0}$ & $\mathbf{3 0}$ & $\mathbf{4 0}$ & $\mathbf{5 0}$ & $\mathbf{6 0}$ \\
\hline PV capacity on low voltage grid [GWP] & 2 & 8 & 15 & 20 & 24 & 30 & 40 \\
PV capacity on M/H voltage grid [GWP] & 1 & 2 & 5 & 10 & 16 & 20 & 20 \\
\hline Grid injection level of PV [kW/kWP] & $\mathrm{l}$ & 0.5 & 0.4 & 0.3 & 0.25 & 0.20 & 0.15 \\
max. PV injection on low voltage grid [GW] & 2 & 4 & 6 & 6 & 6 & 6 & 6 \\
max. PV injection on M/H voltage grid [GW] & $\mathrm{I}$ & 1 & 2 & 3 & 4 & 4 & 3 \\
Net battery capacity on PV system [kWh/kWP] & $\mathrm{n} / \mathrm{a}$ & 0 & 0 & 0.5 & 1.0 & 1.5 & 1.5 \\
Night injection level of battery [kW/kWP] & $\mathrm{n} / \mathrm{a}$ & 0 & 0 & 0.045 & 0.09 & 0.135 & 0.135 \\
Max. night battery injection on grid [GW] & $\mathrm{n} / \mathrm{a}$ & 0 & 0 & 1.35 & 3.6 & 6.75 & 8.1 \\
\hline Share of renewable energy (SRE) [\%] & 10.1 & 27.3 & 47.5 & 54.7 & 62.2 & 69.1 & 72.3 \\
Fraction of RE curtailed (FRC) [\%] & 0 & 3 & 9 & 14 & 18 & 20 & 26 \\
\hline
\end{tabular}


Table V: Proposal for deployment of renewable energy on the Belgian electricity grid simulated for electrical consumption data and availability factors of wind and PV in the year 2015. Overview of the potential of curtailed Renewable Energy connected to the LV or MVIHV grid.

\begin{tabular}{|l|c|c|c|c|c|c|c|}
\hline Proposed phase of deployment & 2016 & 1 & 2 & 3 & 4 & 5 & n/a \\
\hline Wind capacity offshore [GW] & 0.7 & 2.2 & 4 & 4 & 4 & 4 & 4 \\
Wind capacity onshore [GW] & 1.5 & 3 & 5 & 5 & 5 & 5 & 5 \\
\hline Total PV capacity [GWp] & $\mathbf{3}$ & $\mathbf{1 0}$ & $\mathbf{2 0}$ & $\mathbf{3 0}$ & $\mathbf{4 0}$ & $\mathbf{5 0}$ & $\mathbf{6 0}$ \\
\hline PV capacity on low voltage grid [GWP] & 2 & 8 & 15 & 20 & 24 & 30 & 40 \\
PV capacity on M/H voltage grid [GWP] & 1 & 2 & 5 & 10 & 16 & 20 & 20 \\
\hline Share of renewable energy (SRE) [\%] & 10.1 & 27.3 & 47.5 & 54.7 & 62.2 & 69.1 & 72.3 \\
Fraction of RE curtailed (FRC) [\%] & 0 & 3 & 9 & 14 & 18 & 20 & 26 \\
\hline Share of PV injected [\%] & 4 & 11 & 20 & 29 & 38 & 47 & 52 \\
Fraction of curtailed PV [\%] & 0 & 7 & 15 & 19 & 18 & 18 & 25 \\
Share of wind injected [\%] & 6 & 16 & 27 & 26 & 24 & 21 & 20 \\
Fraction of curtailed wind [\%] & 0 & 0 & 4 & 8 & 15 & 23 & 27 \\
\hline Fraction of RE curtailed on LV grid [\%] & 0 & 2.5 & 5 & 7 & 7 & 7.5 & 12 \\
Fraction of RE curtailed on MV/HV grid [\%] & 0 & 0.5 & 4 & 7 & 10.5 & 12 & 13.5 \\
\hline
\end{tabular}

